\title{
Influencia del fraude académico en la formación profesional: las percepciones de estudiantes
}

\author{
Zeneida Ceballos Villada ${ }^{1}$
}

\begin{abstract}
Resumfen
El presente artículo fundamenta en los resultados de una investigación etnográfica denominada "Actitudes frente al fraude académico en ámbitos universitarios", desarrollada en la ciudad de Pasto. Para la investigación se hace uso de diferentes técnicas de recolección de informacion como la entrevista en profundidad, el muro y cuestionario; se analiza la informacion con el metodo destilar la informacion y se obtienen conclusiones que contrastan los hallazgos de la investigación con los resultados de investigaciones con gran reconocimiento nacional e internacional, en las que advierte la influencia desfavorable del fraude en la calidad de los procesos educativos, por cuanto se encuentran percepciones favorables en cuanto al fraude académico.
\end{abstract}

Patabras clave: Fraude académico, pensamientos, formación académica, plagio.

1 Docente Auxiliar, adscrita a la Escuela de Ciencias Sociales Artes y Humanidades de la Universidad Nacional Abierta y a Distancia (UNAD) Colombia. Psicóloga Universidad de Nariño. Especialista en Gerencia de la promoción de la salud y prevención de la enfermedad. Magíster en Docencia Universidad de la Salle, Estudiante de doctorado en Psicología Universidad Maimónides (Argentina).Correo electrónico: zeneida .ceballos@unad.edu.co. 


\title{
ZenédaChallosYllada
}

Influencia del fraude académico en la formación profesional: las percepciones de estudiantes, artículo producto

de la Investigación

\section{The influence of academic cheating in professional formation: students' perceptions}

\begin{abstract}
This article is based on the results of ethnographic research entitled: "Attitudes towards academic fraud in university" developed in the city of Pasto. "This research used different data collection techniques such as in-depth interviews, write-in-the-board and questionnaires. This information was analyzed with the method called "distill information". Conclusions derived from this method offer contrasting results with nationally and internationally reputed research studies, which warn about adverse effects fraud may have in the quality of educational processes, mostly in the scenario of a favorable opinion toward academic cheating.
\end{abstract}

Keywords: Academic cheating, thinking, academic education, plagiarism.

Recibido: 5 Enero de 2011

Aceptado: 16 Marzo de 2011

\section{Introducción}

En el artícul o quesepresentaa continuación se refl exiona sobre los pensamientos de los estudiantes acerca de la influencia que tiene el fraude academico en la formacion, profesional, partiendo de los resultados de investigacion de Ceballos y V ásquez (2009) denominada "A ctitudes frente al fraude academico universitario en ambitos universitarios". Para iniciar es importante tener en cuenta que el fraude academico se ha constituido en un fenomeno cotidiano que lesiona la legitimidad de los procesos eval uativos, pone en riesgo el desempeno académico y el futuro profesional de los psicologos en formacion. Desde la perspectiva de los estudiantes, la tematica es poco explorada e ineludiblemente controversial, Por esta razón, se consideró necesario develar realidades que, aunque cotidianas, resul tan desconocidas.

La investigación, se inscribió, en el paradigma cualitativo, con un enfogue histórico hermenéutico y un método etnográfico. Como técnicas de recoleccion de informacion se emplearon entrevistas en profundidad, cuestionarios y una 
Revista de

investigaciones UNAD

Volumen 10. Número 1. Junio 2011

técnica novedosa denominada "el muro" La información recolectada fue registrada en diario de campo y se analizó mediante la técnica de "destilar la información", que permite analizar la información de manera inductiva logrando garantizar su confiabilidad. La unidad de anal isis estuvo conformada por estudiantes de los programas de psicologia de la Universidad M ariana y la Institucion Universitaria CESM AG de diferentes semestres académicos.

Los resultados de la investigación muestran que existen numerosas formas de fraude y que se presentan especialmente en los examenes. A demas se concluye que el fraude academico surge principalmente de procesos evaluativos inadecuados, de la postura autoritaria de los docentes y del escaso compromiso de los estudiantes y que el fraude, segun la percepcion de los estudiantes, influye de manera desfavorable como favorable en su formacion profesional.

\section{Metodología}

Para investigar acerca de la influencia que tiene el fraude académico en su formacion profesional se partio de un trabajo investigativo que estuvo orientado desde el paradigma cualitativo, con un enfogue hermeneutico y un tipo de estudio etnografico. La investigacion, por sus caracteristicas metodologicas, se inscribio como una investi gacion exploratoriay descriptiva porque se busco comprender en profundidad las actitudes de los estudiantes frente al fenomeno en estudio.

En cuanto a la unidad de análisis, se consideró a los estudiantes de los Programas de Psicología de la Universidad M ariana y de la Institucion UniversitariaCESMA G. Se real izo un muestreo intencional y se utilizó como técnicas de recoleccion de información como la entrevista en profundidad, el muro y el cuestionario.

Finalmente, como instrumento de registro de información se empléo el diario de campo y para el procesamiento y analisis de información se siguilo el metodo Destilar la Información propuesto por Fernando Vásquez Rodríguez (2007), para finalmente llegar a la interpretación correspondiente a partir de campos categoriales técnicamente, conformados, en una dinamica de explicacion comprension y triangulación, con el objetivo de ser coherente y fieles a los hall azgos. En la interpretacion se ponen en juego tres vertices fundamentales: el verricice de la realidad (constituido por la descripcion), e) vértice teorico y el vertice del observador - investigador, este proceso en si mismo, recibe el nombre de triangulación interpretativa y es el que permite aportar, desde la investigación, al conocimiento de las ciencias sociales (De Tezanos, 2001). 


\section{ZenédaCedollosVillada}

Influencia del fraude académico en la formación profesional: las percepciones de estudiantes, artículo producto

de la Investigación

\section{Resultados y discusión}

El comportamiento defraudador es constante en los procesos evaluativos en la educación superior y su presencia es reconocida por docentes y al umnos. Al hacer la comparacion con los hall azgos de otras investigaciones, como la real izada por Eckstein (2003) con el sello de Unesco, o as realizadas desde la Univerśjad de Los Andes en Colombia (M ejía, I. \& Ordoñez, C., 2003 y Ordoñez, Mejla \& Castell lanos, 2006), se constata que el fraude académico universitario se encuentra vigente en diferentes contextos y usando modalidades similares y su uso es generalizado en las instituciones educativas al rededor del mundo. A demas genera costos de diverso orden, como el emocional, relacional, académico y economico, incidiendo en la calidad de la educacion superior.

Entre los instrumentos más reconocidos y usados para hacer fraude académico se encuentran los populares chancucos, chanchullos o papelitos. Estos son tan utilizados en el ámbito académico que es reconocido en el diccionario de la Real A cademia de la Lengua (2007).

De otra parte, los avances en la tecnología han aportado a los alumnos herramientas para el desarrollo de creativas y novedosas formas de fraude. Entre estas se encuentran la utilizacion del Internet como forma de realizar plagio. El uso del Internet se ha extendido a nivel mundial conllevando a que os alumnos tienen la oportunidad de acceder a informacion, de cualquier parte del mundo, y se suele considerar "facil" el atribuirse la autoría de publicaciones de ilimitada procedencia.

En un primer momento se pensó en la Internet como un instrumento pedagógico que posibilitaría el intercambio cultural y de conocimientos, como en realidad lo es. Lo preocupante es la implicacion ética que tiene el uso indebido de la información y el favorecer el encubrimiento de conductas que no deberian ser aceptadas socialmente, pero que al momento está tan difundida que se manifiesta confusion en reconocer que es 10 adecuado o no, a la hora de utilizar informacion de otros autores. Esta situacion se complica aun mas cuando se reconoce la enorme cantidad de publicaciones que se realizan alrededor del mundo dia a dia, y darse cuenta de que resulta muy difícil para el docente identificar los avances en el conocimiento. Estas situaciones incrementan la probabilidad de ocurrencia del fraude, en tanto son mas fáciles las oportunidades para que los alumnos manipulen las eval uaciones a su favor, presentando como suyos trabajos que contienen segmentos de información copiados y pegados desde textos sin la correspondiente referenciacion.

El fraudesepresenta en una gran cantidad deactividades eval uativas, especial mente en aquellas que encuentren mediadas por el conocimiento memoristico y repetitivo. Es importante hacer notar que también se encuentran evidencias de fraude académico en toda la formacion profesional: es decir, se empieza con 


\section{Revista de}

investigaciones UNAD

Volumen 10. Número 1. Junio 2011

el fraude en los primeros semestres y se va con este comportamiento hasta los semestres finales, pero es relevante que los comportamientos fraudulentos tienden a disminuir con el paso de los semestres. En consecuencia, hay una tendencia a presentar mas eventos de fraude en los primeros semestres y menos en los ullimos. Esta situacion se da probablemente porque el nivel de desarrollo va cambiando igual que el tipo de responsabilidades académicas. Por tanto, el sentido de responsabilidad que se adquiere, ante la inminencia de la vida profesional y laboral, es un factor relevante.

El fraude se presenta en diferentes modalidades de evaluación. Sin embargo es en los examenes y especialmente en aquellos en los que el objetivo primordial es la repeticion de contenidos, donde se hace más evidente el comportamiento fraudulento.

Lo anterior permite analizar dos situaciones particulares:: la primera es que los docentes utilizan predominantemente al examen como instrumento de evaluacion, pese a que se encuentran serios cuestionamientos acerca de la validez y confiabil idad para eval uar procesos de formacion y que sus ventajas son limitadas Florez Ochoa (2001). En segunda instancia, el examen en las instituciones de educacion superior, se asume como el, principal indicador de aprendizajes, y por esta razon, se mencionan a los "exámenes parciales, finales y exámenes de habilitaciones" como los momentos eval uativos en donde mas se hace fraude.

En consecuencia, las técnicas evaluativas con un objetivo predominantemente sumativo ponen en tela de juicio la calidad de los procesos educativos y es "alarmante el hecho de que una mala evaluacion puede deteriorar un programa social y ocasionar perjuicios a toda una clase social" (House, 2001. p.13).

En este sentido, pensar en que el uso de fraude académico en sus diferentes modali dades puede influir en la formacion academica y el desempeno profesional de los estudiantes que lo practican haría pensar que es una obviedad. Pero mas alla de ello, los estudiantes tienden a pensar que no siempre es de esa manera por cuanto desligan el exito laboral del rendimiento academico y por tanto, a influencia sería incipiente. Pero al adentrarse en la manera como se concibe la influencia se deben diferenciar dos posturas frente al tema: en la primera se afi irma que la influencia del fraude es favorable y se considera como una forma de crear y fortalecer las relaciones sociales, y la otra es que la influencia del fraude es desfavorable en cuanto afecta el redimiendo academico, la eficacia laboral y algunas situaciones personales.

Cuando se presenta que la influencia del fraude es favorable se privilegian las relaciones interpersonales a la hora de conseguir conquistas laborales, las ubica casi por encima de las competencias que el estudiante o protesional tenga a a hora desempenarse en un trabajo y se ejemplifican con afirmaciones como la siguiente." Para mí el éxito laboral...Depende de las competencias que uno ha 


\section{ZenédaCedollosVillada}

Influencia del fraude académico en la formación profesional: las percepciones de estudiantes, artículo producto

de la Investigación

desarrollado a lo largo de toda su vida y las competencias sobre todo de tipo laboral y las competencias y los valores que uno tiene y entre esos me he dado cuenta $[\mathrm{de}$ que las personas efectivas son aquellas que tienen muy buenas relaciones personales, porque nuestro medio laboral es político y en ese medio político se mueven los poderes de influencia y de relaciones" (Entrevista con estudiante).

En conclusión, el fraude académico, en la percepción de los estudiantes, no tendria repercusiones significativamente desfavorables en el momento actual ni en el futuro. A traves del fraude se construyen redes sociales importantes que son de larga duración y que por el contrario potencializan oportunidades futuras. Y ll egaría a ser sobreval orado en a medida que es visto como una forma de expresion creativa:" "La trampa es un acto de inteligencia y creatividad, sumale la ética Creatividad + etica (Informacion del M uro)"

De esta manera, pareciera que el asunto ético es relegado o trivializado, realmente priman los motivos personales para superar una calificacion por tanto, una estrategia de supervivencia académica: "(frente al hecho de hacer fraude) despues se convierțe en un aprendizaje... Cuando me siento a pensar como hago para que la proxima vez no me pilleen.... entonces ya no lo veo como antiético, sino como una alternativa de solucion" (entrevista estudiantes).

Pero por otra parte, estudiantes cuestionan el fraude en términos de "justicia" y la necesidad de ganar satisfaccion al al canzar logros por, méritos, y en este sentido el fraude influye en la reafirmacion de la limitación en posibilidades o capacidades para obtener, conquistas académicas y por tanto, la valoracion personal de si mismo se vería deteriorada. A demas, se encontro que en sintonía con Garcia y M achado (2004), una mayor percepción de los estudiantes acerca del fraude en la clase y una menor conciencia de la gravedad del mismo, vendrian acompanadas de, una probabilidad superior de cometer fraude en exámenes, asi como en exámenes y trabajos conjuntamente.

Es así como el asunto de lo ético puede por momentos generar conflictos internos en los que se autocuestiona acerca de las posturas personales en las que se llega afirmar "(referente al fraude)... está tan ligado a tener etica, se vuelve de doble moral y, dice, yo estudio psicología, todas las personas l consideran la persona más correcta, que no va a hacer fraude... para mi hablar de etica o no tener etica se convierte como un eterno dilema" (entrevișta estudiantes). Si bien se reconocen implicaciones eticas por el prestigio social que tiene o tendrá su rol profesional, se presenta una disonancia interesante: por un lado se muestra como una actividad normal que niega la influencia de o ético, pero a la vez propone estrategias para superarlo a través del cambio de las estrategias eval uativas en definitiva, la responsabilidad, se delega en gran parte a las estrategias eval uativas util izadas por parte de los docentes y tutores. 
Revista de

investigaciones UNAD

Volumen 10. Número 1. Junio 2011

En consecuencia, se está trabajando acerca de uno de los componentes fundamental es dentro del proceso pedagogico que es: evaluacion. Si se jnicia con el concepto de evaluación se tiene que la comprensión de la eval uacion del aprendizaj e puede darse como comunicacion y es vital para entender por que sus resultados no dependen sólo de las caracteristicas del "objeto" que se evalua, sino, ademas, de las pecul iaridades de quien, (es) reaaliza (n) la evaluacion y de los víncul os que establezcan entre sI (Gonzal ez y Perez, 2006); pero a traves de los hall azgos de la investigacion se encuentra que el concepto de eval uacion está ligado a una concepcion tradicional, en la que es sinoni mo de calificacion y en la que se establ ece como una relación jerárquica en la que el docente tiene el poder. La evaluacion para buena parte de los estudiantes es una oportunidad de hacer fraude.

La finalidad de la evaluación develada desde diferentes posturas conceptuales, es mejorar los procesos y resultados académicos y por tanto busca: diagnosticar el nivel de desarrollo de los estudiantes, asegurar el éxito del proceso educativo, identificar los estilos personales de aprendizaje, identificar dificultades, deficiencias y limitaciones, ofrecer oportunidades; afianzar los aciertos y corregir oportunamente los errores; promover, certificar o acreditar a los estudiantes, en conclusion orientar el proceso educativo y mejorar su calidad; pero a traves de la investigacion se puede resignificar en cuanto se encuentra que la finali dad de la eval uacion es percibida como medir y clasificar a los estudiantes. El fin, principal aprobar o superar una prueba, comprobar de modo sistematico en qué medida se ha logrado los resultados previstos en los objetivos que se hubieran especificado antelación. "Es la forma mediante la cual medimos y juzgamos el aprendizaje con el fin de certificarlo, asignar calificaciones determinar proporciones etc." (Carreño, 1985, tomado por M onedero 1998 p. 41).

De esta manera, se puede afirmar que el fraude en cuanto a lo negativo no influye significativamente en la formacion de los estudiantes, por el contrario, los estudiantes consideran que en ciertas situaciones posibilita el establecimiento de relaciones sociales que pueden ser duraderas, pero tambien pueden llevar a desconfiar de sus propias habilidades, aunque desde la teoria como la planteada por Cesar Coll $(1983$, p. 16) se tiene que la evaluacion es la que tiene lugar durante el desarrollo del, proceso educativo y, sobre todo en la medida que proporcione indicadores útiles para reconduciril $0^{\prime \prime}$.

\section{Conclusiones}

La eval uación de aprendizaje, es sin duda un proceso complejo "La escala a la que se ll eva a cabo las activi dades de eval uacion su omni presencia y diversidad hace difícil su comprension, incluso a los que se mueven en este campo" (House, 


\section{ZenédaCedollosVillada}

Influencia del fraude académico en la formación profesional: las percepciones de estudiantes, artículo producto

de la Investigación

2011, p. 13). En este sentido, el comportamiento defraudador es constante en los procesos evaluativos en la educación superior y su presencia es reconocida por docentes y alumnos. Al hacer la comparación con los hallazgos de otras investigaciones como la realizada por Eckstein (2003) o las realizadas desde la Universidad de Los Andes en Colombia (2003 y 2005) se tiene que el fraude academico universitario se encuentra vigente en diferentes contextos y usando modalidades similares y su uso es generalizado en las instituciones educativas al rededor del mundo. A demas, genera costos de diverso orden como el emocional, relacional, academico y económico, incidiendo en la calidad de la educacion superior.

Entre los instrumentos más reconocidos y usados para hacer fraude académico se encuentran los populares chancucos, chanchullos o papelitos. De otra parte, los avances en la tecnologia han aportado a los alumnos herramientas para el desarrollo de creativas y novedosas formas de fraude, entre estas se encuentran la utilizacion del Internet, como forma de realizar plagio.

En un primer momento se pensó en el Internet como un instrumento pedagógico que posibil itaria el intercampio cul tural y, de conocimientos, como en real idad lo es, lo preocupante es la implicación ética que tiene el uso indebido de la información, el favorecer el encubrimiento de conductas que no deberían ser aceptadas socialmente, pero que al momento esta tan difundida que se manifiesta confusion en reconocer que es 10 adecuado o no, a la hora de utilizar información de otros autores.

En cuanto a los pensamientos de los estudiantes en torno al fraude, se encyentra que, tanto docentes como alumnos consideran que el fraude academico universitario no es del todo un riesgo para el buen desempeño academico y profesional presente o futuro, por el contrario se cree que el comportamiento defraudador es un indicador de la capacidad de interrelacionarse del alumno y que esa capacidad desarrollada contribuye en los proyectos profesionales 0 laborales futuros.

Con 10 anterior se puede evidenciar que para que se presenten eventos de fraude influyen varios factores: los factores psicologicos adquieren preponderancia en la medida que permiten vislumbrar como algunas ideas irracionales sobre la propia eficacia y la propia capacidad hacen que el estudiante genere inseguridad frente a su proceso de aprendizaje y opte por el fraude como una estrategia para solventar el déficit personal evaluado.

Por otro lado, a nivel académico, se presenta una importante dificultad en cuanto a las técnicas de eval uacion que se utiliza, las pruebas, son esencialmente escritas y se caracterizan por ser memoristicas y de caracter acumulativo, es decir se realizan evaluaciones sumativas, en donde los procesos y el aprendizaje quedan en un segundo plano y se asume la evaluacion como un 


\section{Revista de \\ investigaciones UNAD \\ Volumen 10. Número 1. Junio 2011}

proceso más administrativo y sancionatorio más que formativo. Lo anterior, sumado a la actitud represiva y dominante del docente se convierte en estimul os favorecedores del fraude. Ademas, la participación en el fraude ya sea para beneficio personal o para beneficiar a los otros se considera como parte de la adaptacion del al umno al grupo academico del cual hace parte.

\section{Referencias bibliográficas}

Bogoya, D. (2006). Evaluación educativa en Colombia. Seminario Internacional de Evaluación. Cartagena de Indias, 16 y 17 de febrero de 2006. Bogotá: Icfes.

De Tezanos, Araceli. (2001). Una etnografía de la etnografía. Bogotá: Anthropos.

Flórez Ochoa, Rafael y Tobón Restrepo, Alonso. (2001) Investigación Educativa y pedagógica, Bogotá: Mc Graw Hill. .

García, E. y Machado, B. (2005). Un modelo econométrico del fraude académico en una universidad española. Universidad Complutense de Madrid. [Internet]. Disponible en: http:/www.ucm.es/BUCM/cee/doc/9820/9820.htm

House, E. (2000). Evaluación ética y poder. Madrid: Morata.

Mejía, José Fernando y Ordoñez, Claudia.(2003) El fraude en la Universidad de los Andes ¿Qué, Qué tanto y por qué?. Revista de Estudios Sociales No. 18

M ejía, R. (2006). Educación(es) en las(s) globalización(es) I. Entre el pensamiento único y la nueva crítica. Bogotá: Desde Abajo.

Real Academia Española. (2005). Diccionario Panhispánico De Dudas Bogotá: Santillana.

Real Academia de la lengua española (2007). Diccionario de la lengua española. Universidad Complutense de Madrid. [Internet]. Disponible en: http://www .rae.es/

Vásquez, Fernando. (2007) Destilar la información, trabajo presentado en la I.U.CESM AG, Pasto - Nariño - Colombia. 